\title{
Erratum
}

\section{Erratum to "Associations of Cardiorespiratory Fitness and Fatness with Metabolic Syndrome in Rural Women with Prehypertension"}

\author{
Patricia A. Hageman, ${ }^{1}$ Carol H. Pullen, ${ }^{2}$ Melody Hertzog, ${ }^{3}$ \\ Linda S. Boeckner, ${ }^{4}$ and Susan Noble Walker ${ }^{2}$ \\ ${ }^{1}$ Division of Physical Therapy Education, School of Allied Health Professions, College of Medicine, \\ University of Nebraska Medical Center, Omaha, NE 68198-4420, USA \\ ${ }^{2}$ College of Nursing, University of Nebraska Medical Center, Omaha, NE 68198-5330, USA \\ ${ }^{3}$ College of Nursing, University of Nebraska Medical Center, Lincoln, NE 68588-0220, USA \\ ${ }^{4}$ Nutrition and Health Sciences, University of Nebraska-Lincoln, Lincoln, NE 68583-0806, USA \\ Correspondence should be addressed to Patricia A. Hageman; phageman@unmc.edu
}

Received 27 February 2014; Accepted 30 March 2014; Published 28 April 2014

Copyright (C) 2014 Patricia A. Hageman et al. This is an open access article distributed under the Creative Commons Attribution License, which permits unrestricted use, distribution, and reproduction in any medium, provided the original work is properly cited.

In the original paper, the authors discovered a computer coding error that resulted in 33 of the women's ages being incorrectly recorded.

All analyses were repeated for this paper using the corrected age dataset, as all our logistic regression analyses in the published paper were adjusted for age. The repeated analyses, using the corrected dataset, lead to minor changes that needed to be reported to the results in the published paper. These corrections did not change the conclusion of the published paper. The authors apologize for any inconvenience.

In the Abstract. line 8, should be changed from $7 \%$ to $8 \%$; line 9 , should be changed from $75 \%$ to $81 \%$; line 9 , should be changed from $59 \%$ to $69 \%$.

Tables 2-3 were updated as provided.

In Section 2.1. page 3, column 1, paragraph 2, line 1, $57.8 \pm 7.6$ should be changed to $56.4 \pm 6.3$.
In Section 2.2. page 3, column 1, paragraph 1, line 5, 70 should be changed to 170 .

In Section 3. page 4, column 2, paragraph 2, line 5, 1.21 should be changed to 1.20 ; in line $5,1.14-1.29$ should be changed to 1.13-1.27; in line $8,7 \%$ should be changed to $8 \%$; in line 9 , 0.93 should be changed to 0.92 ; in line $9,0.90-0.97$ should be changed to $0.88-0.96$; in line $10,=0.001$ should be changed to $<0.001$.

In Section 3. page 4, column 2, paragraph 3, line 5, 75\% should be changed to $81 \%$; in line $6,0.25$ should be changed to 0.19 ; in line $6,0.12-0.52$ should be changed to $0.09-0.41$; in line $10,59 \%$ should be changed to $69 \%$; in line $10,0.41$ should be changed to 0.31 ; in line $10,0.19-0.87$ should be changed to $0.14-0.69$; in line $10,0.02$ should be changed to 0.004 . 
TABLE 2: Logistic regressions predicting the metabolic syndrome from percent body fat and estimated cardiorespiratory fitness.

\begin{tabular}{|c|c|c|c|c|c|c|c|c|c|c|c|c|}
\hline & \multicolumn{4}{|c|}{ Model 1} & \multicolumn{4}{|c|}{ Model 2} & \multicolumn{4}{|c|}{ Model 3} \\
\hline & $b$ & OR & $95 \%$ CI & $P$ & $b$ & OR & $95 \% \mathrm{CI}$ & $P$ & $b$ & OR & $95 \% \mathrm{CI}$ & $P$ \\
\hline$\%$ Body fat & 0.18 & 1.20 & $1.13-1.27$ & $<0.001$ & 0.20 & 1.22 & $1.13-1.31$ & $<0.001$ & 0.07 & 1.07 & $0.88-1.30$ & NS \\
\hline Fitness & & & & & 0.02 & 1.02 & $0.96-1.08$ & NS & -0.24 & 0.78 & $0.53-1.15$ & NS \\
\hline$\%$ Body fat $\times$ fitness & & & & & & & & & 0.01 & 1.01 & $1.00-1.01$ & NS \\
\hline Fitness & -0.09 & 0.92 & $0.88-0.96$ & $<0.001$ & & & & & & & & \\
\hline
\end{tabular}

All models were adjusted for age, education, and household income.

Nagelkerke $R$ squares were $0.24,0.24$, and 0.25 for \% body fat models 1,2 and 3 , respectively.

Nagelkerke $R$ square was 0.11 for fitness model 1 .

TABLE 3: Logistic regressions predicting metabolic syndrome by unfit/fat and fit/fat categories using two body composition methods.

\begin{tabular}{lcccc}
\hline & Cases & $b$ & OR & $95 \%$ CI \\
\hline & & Model 1-fit/fat categories defining obesity by percent body fat & \\
Unfit/fat & $78 / 188(41.5 \%)$ & & 1 & $0.09-0.41$ \\
Fit/fat & $12 / 85(14.1 \%)$ & -1.64 & 0.19 & $<0.001$ \\
\hline & & Model 2-fit/fat categories defining obesity by revised BMI cut-score & \\
Unfit/fat & $78 / 179(43.6 \%)$ & & 1 & $0.14-0.69$ \\
Fit/fat & $12 / 57(21.1 \%)$ & -1.17 & 0.31 & 0.004 \\
\hline
\end{tabular}

"Fat" was defined as body fat cut-score $\geq 30 \%$ and as $\geq 25 \mathrm{~kg} / \mathrm{m}^{2}$ for revised BMI obesity cut-score.

"Fit" was $>25 \mathrm{~mL} / \mathrm{kg} / \mathrm{min}$. Both models were adjusted for age, education, and household income.

Both models excluded women classified as "not-fat" as there were no cases of metabolic syndrome in women classified as "non-fat."

Nagelkerke $R$ square was 0.13 and 0.07 for models 1 and 2, respectively. 


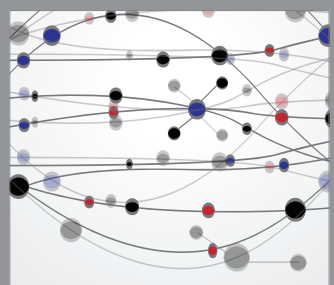

The Scientific World Journal
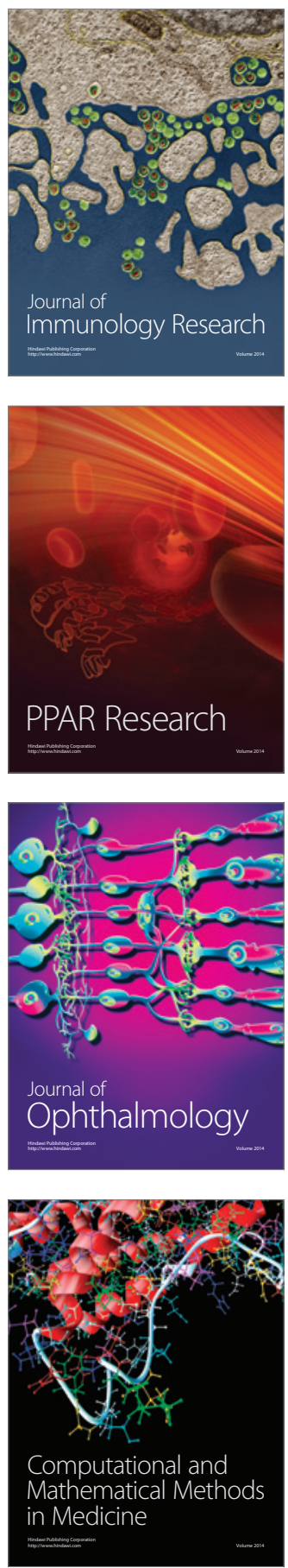

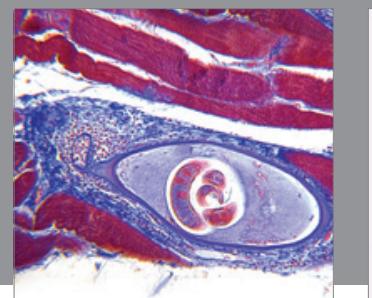

Gastroenterology

Research and Practice
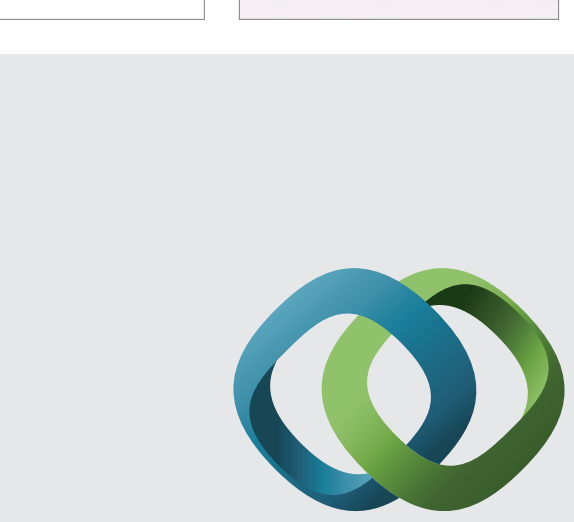

\section{Hindawi}

Submit your manuscripts at

http://www.hindawi.com
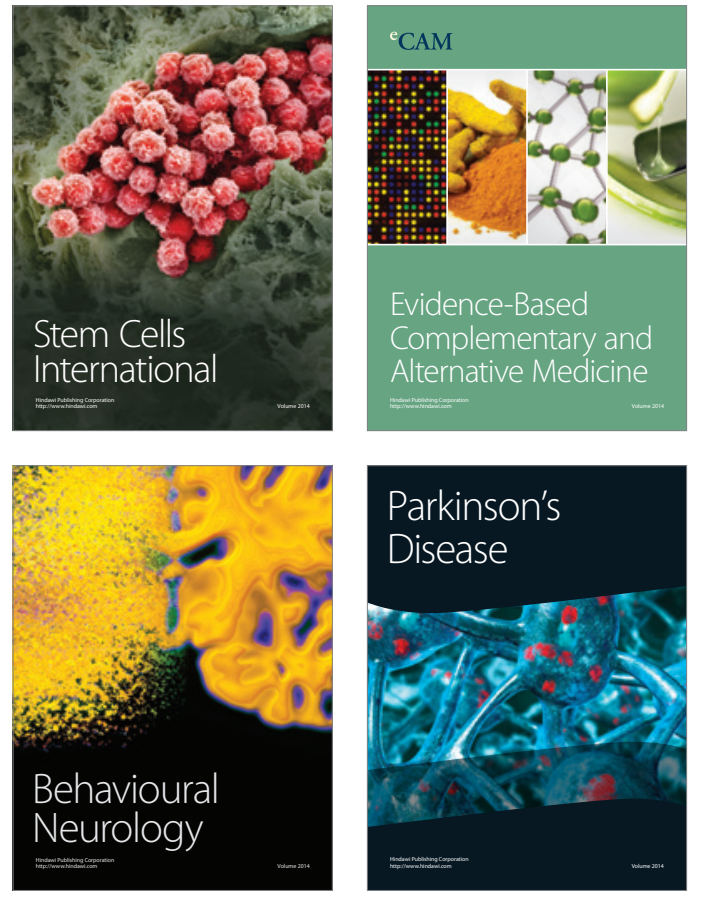
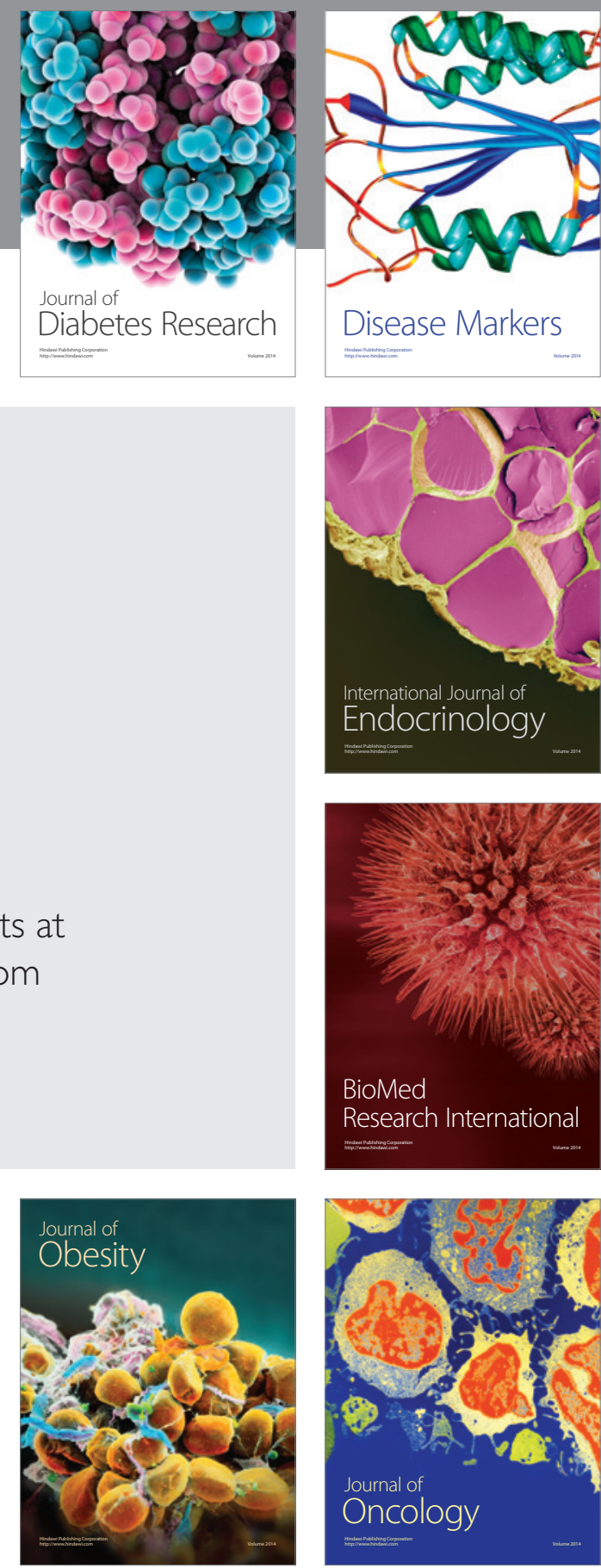

Disease Markers
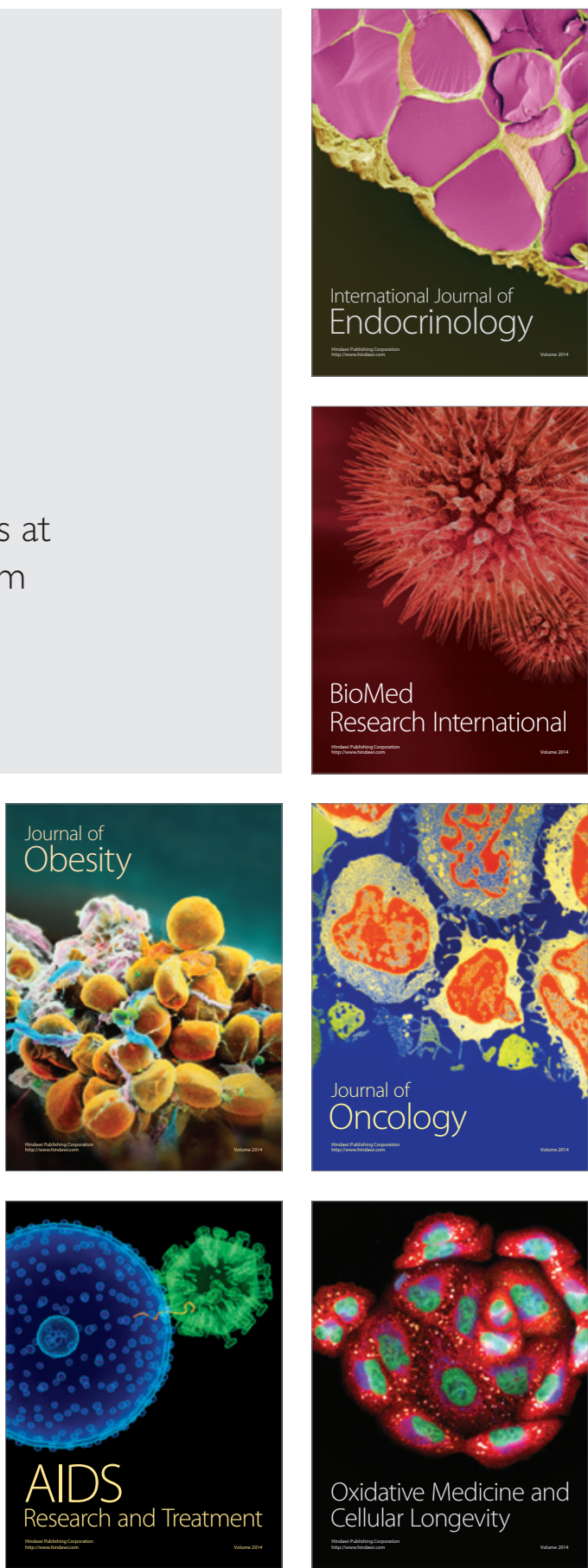